\title{
Efficacy of Glycopyrrolate in Primary Hyperhidrosis Patients
}

\author{
Department of Anesthesiology and Pain Medicine, Ajou University College of Medicine, Suwon, \\ *Kichan Pain clinic, Seoul, Korea
}

Hyun Ho Lee, MD, Do Won Kim, MD, Do Wan Kim, MD, and Chan Kim, MD*

\section{Background:}

Primary hyperhidrosis is a disorder of excessive sweating, which shares several features with anxiety disorders and has a negative impact on a patient's quality of life. Oral glycopyrrolate is one of the treatments available. There are a few published studies on the use of glycopyrrolate given orally in the treatment of hyperhidrosis.

\section{Methods:}

Thies is study was a review of case notes in a series of 36 patients with primary hyperhidrosis. We made a comparison between the Keller's scale score of a pre-glycopyrrolate medication group and the Keller's scale score f a post-glycopyrrolate medication group. The Milanez de Campos score, Short Form_36 (SF-36) score, Beck Depression Inventory (BDI) score, Beck Anxiety Inventory (BAI) score, and autonomic nervous system (ANS) scale score were also compared between the two groups.

\section{Results:}

In the post-glycopyrrolate medication group, there were declines in Keller's scale, and Milanez de Campos scale score and BAI score $(P<0.001)$. In addition, there were increases in SF_36 score in the post-glycopyrrolate medication group $(P=0.03)$ However, no changes were seen in, BDI score and ANS score in the post-glycopyrrolate medication group $(P<0.001)$.

\section{Conclusions:}

Glycopyrrolate is an effective initial method of treating primary hyperhidrosis that, reduces anxiety and improve patients' quality of life. (Korean J Pain 2012; 25: 28-32)

\section{Key Words:}

anticholinergic agents, glycopyrrolate, primary hyperhidrosis. 


\section{INTRODUCTION}

Hyperhidrosis is a condition of abnormal sweating, in which a larger amount of perspiration occurs than is needed to control body temperature. Hyperhidrosis is classified into local or general hyperhidrosis according to sweat secretion.

Hyperhidrosis is also differentiated according to time of onset and cause. Primary hyperhidrosis usually occurs during puberty or earlier and characteristically has a strong genetic tendency. Secondary hyperhidrosis occurs at a much later period and accompanies another disease. It is reported that primary hyperhidrosis shows a very good response to treatment, and various treatment methods are employed [1-4].

The preferred treatment method for primary hyperhidrosis should be one that is noninvasive and simple, with few complications and a low financial burden. In this regard, oral administration of medication can be a good initial treatment method.

This study investigated the effect of glycopyrrolate administration on symptoms of primary hyperhidrosis and other clinical symptoms.

\section{MATERIALS AND METHODS}

\section{Subjects}

Subjects were chosen from patients who visited our pain clinic from January 1, 2007 to December 31, 2009 and were diagnosed with primary hyperhidrosis. Treatment for hyperhidrosis was explained to the patients, and those who decided to take glycopyrrolate were enrolled in the study.

Selection criteria for subjects were as follows: a) age 10 or above and less than 80 , b) diagnosed with hyperhidrosis for the first time in their visit to our clinic, c) primary hyperhidrosis patients with no underlying diseases as confirmed by interview, thyroid function test, electrocardiogram, chest X-ray, and blood tests, d) patients with no experience of invasive treatment in their medical history, e) oral administration of medication confirmed to be possible by exclusion of asthma, dysuria, ophthalmologic disease, and cardiac disease through interview, and f) $\mathrm{pa}^{-}$ tients who were able to fill out questionnaires personally or with help from the investigator.

Prior to beginning the treatment, the effects of the medication, side effects, and warnings were sufficiently explained, and treatment proceeded when consent was obtained.

\section{Method}

Glycopyrrolate $1 \mathrm{mg}$ was administered twice a day as the initial dosage. Then, according to the alleviation of symptoms, the dosage was increased by $2 \mathrm{mg}$ per day up to a maximum of $8 \mathrm{mg}$ per day. If complications that caused difficulties in everyday life arose after increasing the dosage, the dosage was decreased to that directly preceding the current level. The effect and side effects of the medication were examined through weekly questionnaires filled out by the subjects, interviews with the investigator, and physical examinations. The questionnaire included basic information such as age, sex, initial occurrence of hyperhidrosis, physical state, medical history, allergies to medications, underlying disease, family history, and area of perspiration. To examine the improvement of hyperhidrosis symptoms, subjects were given questionnaires including questions from the Keller scale and the Milanez de Campos scale, and the scores were compared. The function of the autonomic nervous system (ANS) was scored through a test comprising 90 questions. In addition to examining the direct symptoms of hyperhidrosis, a Beck Depression Inventory (BDI) [5] and Beck Anxiety Inventory (BAI) [6] were conducted to examine the effect of the treatment on patients' psychology. A Short Form 36 (SF-36) survey was conducted and analyzed before and after administration of medication to examine the changes in quality of life, which reflects the physical and psychological state of the patient [7].

\section{Statistical analysis}

Descriptive statistics were used to obtain the average score of each indicator and analyze the characteristics of the patients, and a paired T-test was used to analyze the changes in the various indicators before and after administration of medication. Since the variables include some that do not follow the normal distribution, a non-parametric analysis was carried out using a Wilcoxon signed-rank test to analyze the changes in the indicators before and after medication. All analysis used SPSS 12.0.1 (SPSS Inc., Chicago, IL, USA) for Windows. Statistical significance was defined as $P<0.05$. 


\section{RESULTS}

66 hyperhidrosis patients filled out the questionnaires before and after medication. Out of these, 18 were $\mathrm{ex}^{-}$ cluded because they omitted one or more questions while completing the before and after questionnaires, and 12 were excluded because outpatient follow-up was not possible. Thus, a total of 36 patients were administered glycopyrrolate and answered all the questions on the questionnaire (Table 1).

The areas where hyperhidrosis occurred most frequently were, in descending order, the hands, feet, axillae, head and neck, perineum, and other. $19.4 \%$ of the patients were confirmed to have symptoms in only one area, while $27.8 \%$ had symptoms in two areas and $52.8 \%$ had symptoms in three or more areas simultaneously.

Table 1. Demographic Date and Patient Characteristics in Study Population

\begin{tabular}{lc}
\hline \multicolumn{1}{l}{ Classification } \\
Sex, male, \% (n) & $41.6 \%(15)$ \\
Age, year & $27.6 \pm 14.9$ \\
Onset age, year & $15.2 \pm 10.9$ \\
Family history, \% (n) & $25.0 \%(9)$ \\
Night sweating, \% (n) & $19.4 \%(7)$ \\
\hline
\end{tabular}

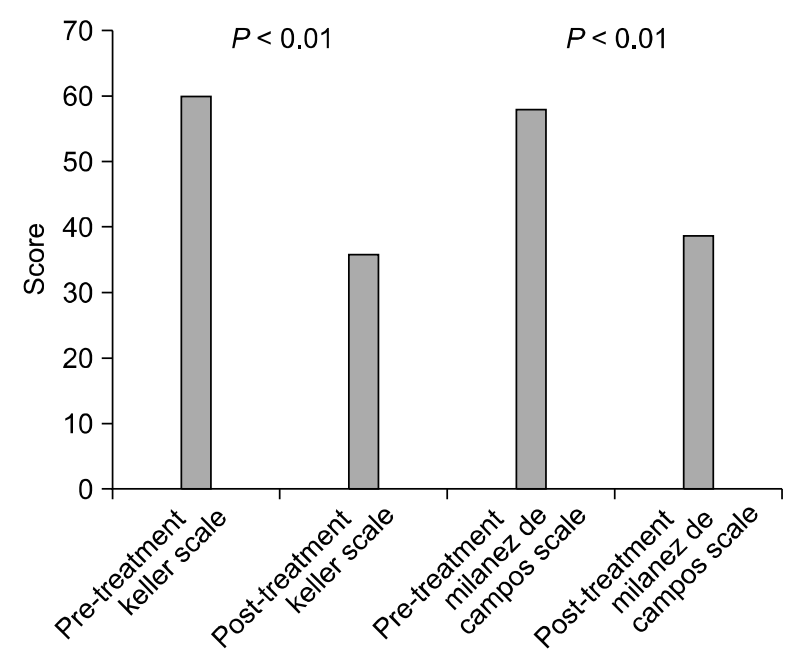

Fig. 1. Comparison of the Keller scale and the Milanez de Campo scale of Pre- and post-glycopyrrolate medication groups.
When comparing the Keller scale before and after administration of glycopyrrolate, $75.0 \%$ of patients showed an actual decrease in perspiration, and a significant improvement was seen, with a mean score before medication of 60 , compared to a mean score after medication of 35.9. It was confirmed that there was an actual decrease in perspiration $(P<0001)$. The Milanez de Campos scale also showed significant change; the mean score of 57.9 before medication improved to 38.7 after medication, providing confirmation that the discomfort level in everyday life had also decreased $(P<0.001)$ (Fig. 1).

BDI and ANS scores did not show large differences before and after medication, but the BAI, which indicates an anxiety disorder, showed a significant decrease when scores before and after administration of medication were compared (change from 12.1 to 9.7, $P=0.03$ ) (Fig. 2).

Table 2. Analysis of Side Effect of Glycopyrrolate

\begin{tabular}{lr} 
Classification & \multicolumn{1}{c}{$\%(n)$} \\
\hline Dry mouth & $27.8 \%(10)$ \\
Palpitation & $11.1 \%(4)$ \\
Headache & $2.8 \%(1)$ \\
Dizziness & $0 \%(0)$ \\
Constipation & $0 \%(0)$ \\
Dysuria & $0 \%(0)$ \\
Others & $8.3 \%(3)$ \\
\hline
\end{tabular}

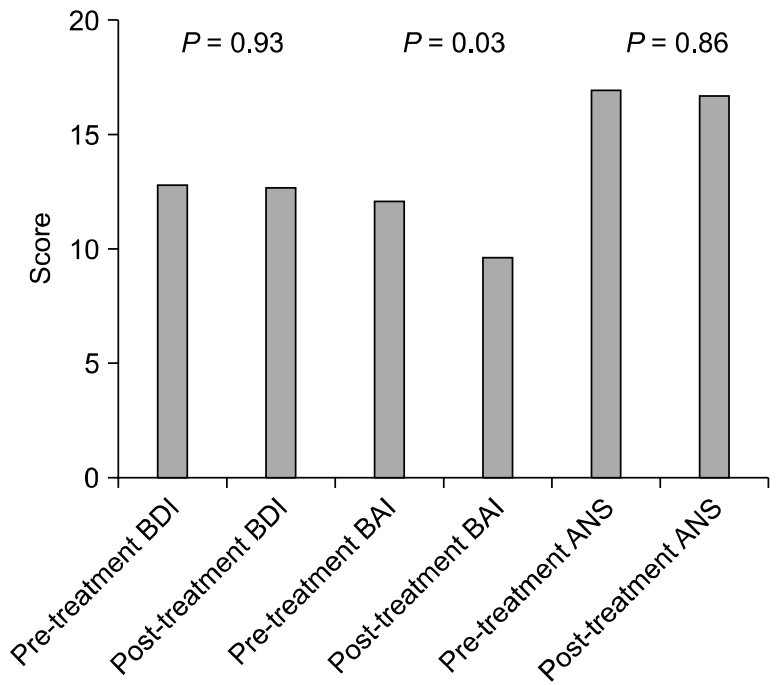

Fig. 2. Comparison of the beck depression inventory (BDI), beck anxiety inventory (BAl) \& autonomic nervous system (ANS) scale of pre- and post-glycopyrrolate medication groups. 
While taking medication, $36.1 \%$ of patients experienced side effects, but none stopped taking the medication due to this cause (0\%). Side effects identified were oral dryness $27.8 \%$, palpitations $11.1 \%$, headache $2.8 \%$, and other $8.3 \%$ (Table 2).

\section{DISCUSSION}

Primary hyperhidrosis has an occurrence rate of 0.6-1.0\% of the entire global population, but among Asians, the rate is as high as $10 \%$, including light symptoms [2]. For a diagnosis of primary hyperhidrosis, it must be confirmed that there is no other disease that could cause the symptoms, which usually appear in areas such as the hands, feet, axillae, trunk, and head and neck. Perspiration is generally induced or intensified by increases in body temperature, exercise, and psychological stimuli. The specific mechanism is not yet known, but symptoms are thought to be due to a hypersensitive reaction of the sympathetic nervous system, and heredity is also thought to play a role $[4,8]$.

Hyperhidrosis hinders the everyday life of many patients. Increase of perspiration due to psychological stimuli is a common phenomenon in the general public, but when the amount of perspiration is excessive, it can hinder the social life of patients, lower their quality of life, and may even result in psychological problems such as depression [4,9]. Therefore, treatment is essential for these patients, and various treatment methods are being attempted.

Typical methods of treatment include topical application of aluminum chloride, ionospheresis, oral medication, hypodermic injection of botulinum toxin, sympathetic nerve block using alcohol, and sympathectomy. These methods each have advantages and disadvantages according to their mechanisms of action. Sympathectomy has an excellent perspiration suppression effect but has serious complications of compensatory sweating, and it has a high failure rate in facial hyperhidrosis [1,10-12]. Hyperhidrosis treatment using botulinum toxin has been frequently used recently, but it has limitations, including that it is effective only in specific areas such as the axillae, and that it is rather expensive $[3,13]$. On the other hand, the rather inexpensive method of topical application of aluminum chloride is also only effective in the axilla area, and it can cause allergic responses in the skin. Ionospheresis is also only effective in specific areas (axillae, hands, feet), the effect is relatively small, and many patients complain of skin rashes and eruption or burning sensation.

Oral medications used in treating hyperhidrosis are sedative or anticholinergic drugs. Oral medication affects the whole body, so there is no compensatory sweating, and it has the advantages of being cheap and easy to take. Sedative drugs are a treatment method that works by suppressing the mechanism by which symptoms appear when the patient is psychologically anxious, but these drugs can cause the side effects of drowsiness and decrease in motivation. Most primary hyperhidrosis patients are young and socially active, so this method of treatment cannot be used in many patients. Anticholinergic drugs act directly on the nerves in the sweat glands to show a perspiration suppression effect [14]. However, anticholinergic drugs suppress the sympathetic nervous system, so symptoms such as oral dryness, palpitations, constipation, dysuria, digestive problems, dizziness, and headaches can occur, and this method cannot be used in patients with underlying diseases such as cataracts or urologic disease. Despite these disadvantages, anticholinergic drugs are still the most frequently selected oral medication. The anticholinergic drug that is used the most often is glycopyrrolate. This drug has fewer central side effects, such as drowsiness or headache, than other anticholinergic drugs, and it has a superior perspiration suppression effect, so it has been widely used overseas as the initial medication in hyperhidrosis treatment [3].

Glycopyrrolate is a drug originally developed for peptic ulcer disease, but it has a perspiration suppression effect, so it is now used frequently in hyperhidrosis treatment. Glycopyrrolate competes with acetylcholine at the muscarinic receptor to cause an anticholinergic effect. In addition, it is a drug in the highly polar quaternary ammonium group and cannot pass through lipid membranes, so it cannot penetrate the brain-nerve barrier and has little effect on the central nervous system. In contrast, atropine sulfate and scopolamine hydrobromide are highly non-polar tertiary amines which can easily pass through lipid membranes [9].

Our study analyzed the effect of orally administered glycopyrrolate in 36 primary hyperhidrosis patients. The study is meaningful in that it analyzed changes in everyday life, psychological state, quality of life, and the autonomic nervous system, in addition to actual decrease of perspiration. Actual decrease of perspiration appeared in $75 \%$ of 


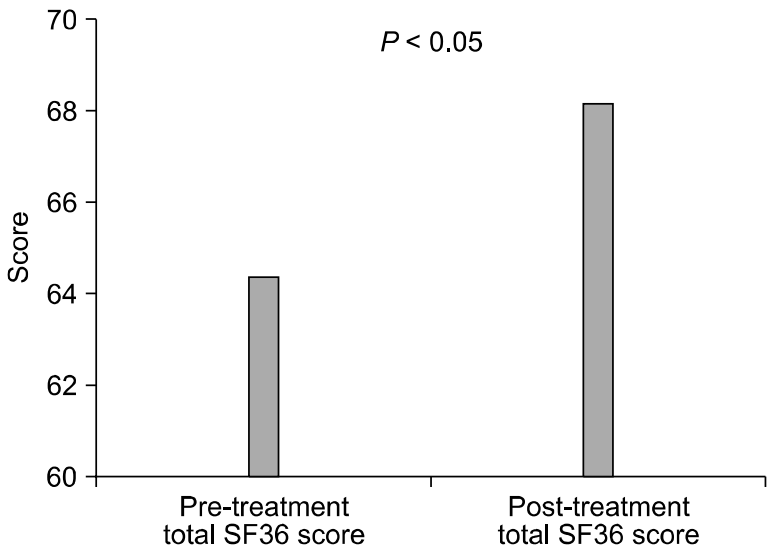

Fig. 3. Comparison of the total short form (SF) 36 score of Pre- and post-glycopyrrolate medication groups.

the patients who used the medication, and decreased level of discomfort in everyday life was also observed. Although changes in depression (BDI) and ANS scores could not be observed, symptoms of hyperhidrosis was suppressed and SF-36 score increased with the BAI score reduced, indicating that the treatment could improve the actual social life of patients (Fig. 1-3).

Of course there are limitations to this study. First, it was difficult to compare the treatment with other medications. This was because of the limited number of $\mathrm{pa}^{-}$ tients and the difficulty in gathering a comparison group administered a different drug, due to ethical reasons. In addition, the study was mainly based on questionnaire analysis, so confirmation of objective results was difficult.

When selecting a treatment method for hyperhidrosis, the current state of the patient and preference of the patient's doctor can be influential. For a permanent effect, an invasive treatment method must be selected, but in general, for safe and reversible initial treatment, medication can be the solution. Therefore, the authors suggest glycopyrrolate as an effective drug that can reduce the degree of hyperhidrosis and is safe and convenient for clinical use.

\section{REFERENCES}

1. Henteleff HJ, Kalavrouziotis D. Evidence-based review of the surgical management of hyperhidrosis. Thorac Surg Clin 2008; 18: 209-16.

2. Klaber M, Catterall M. Treating hyperhidrosis. Anticholinergic drugs were not mentioned. BMJ 2000; 321: 703.

3. Naumann M, Lowe NJ. Botulinum toxin type $A$ in treatment of bilateral primary axillary hyperhidrosis: randomised, parallel group, double blind, placebo controlled trial. BMJ 2001; 323: 596-9.

4. Kim WO, Yoon DM, Kil HK, Kim Jl, Choi Bl. Effect of oral glycopyrrolate in essential hyperhidrosis. Korean J Anesthesiol 2004; 46: 298-301.

5. Craven JL, Rodin GM, Littlefield C. The Beck Depression Inventory as a screening device for major depression in renal dialysis patients. Int J Psychiatry Med 1988; 18: 365-74.

6. Leyfer OT, Ruberg $\mathrm{J}$, Woodruff-Borden J. Examination of the utility of the Beck Anxiety Inventory and its factors as a screener for anxiety disorders. J Anxiety Disord 2006; 20: 444-58.

7. McHorney CA, Ware JE Jr, Raczek AE. The MOS 36-Item Short-Form Health Survey (SF-36): II. Psychometric and clinical tests of validity in measuring physical and mental health constructs. Med Care 1993; 31: 247-63.

8. Ro KM, Cantor RM, Lange KL, Ahn SS. Palmar hyperhidrosis: evidence of genetic transmission. J Vasc Surg 2002; 35: 382-6.

9. Ruchinskas R. Hyperhidrosis and anxiety: chicken or egg? Dermatology 2007; 214: 195-6.

10. Bugmann P, Robert J, Magistris M, Le Coultre C. Thoracoscopic sympathectomy using ultrasonic coagulating shears: a technical improvement in the treatment of palmar hyperhidrosis. Pediatr Surg Int 2002; 18: 746-8.

11. Reisfeld R. One-year follow-up after thoracoscopic sympathectomy for hyperhidrosis. Ann Thorac Surg 2007; 83: 358-9.

12. Adams BB, Vargus-Adams JN, Franz DN, Kinnett DG. Hyperhidrosis in pediatric spinal cord injury: a case report and gabapentin therapy. J Am Acad Dermatol 2002; 46: $444-6$.

13. Wollina $U$, Karamfilov $T$, Konrad $H$. High-dose botulinum toxin type A therapy for axillary hyperhidrosis markedly prolongs the relapse-free interval. J Am Acad Dermatol 2002; 46: 536-40.

14. Ali-Melkkilä T, Kanto J, lisalo E. Pharmacokinetics and related pharmacodynamics of anticholinergic drugs. Acta Anaesthesiol Scand 1993; 37: 633-42. 